\title{
Carga económica de la varicela en niños en Perú, entre 2011 y 2016
}

\author{
Economic Burden of Varicella in Children in Peru from 2011 to 2016 \\ María Esther Castillo ${ }^{1,2, a}$, Raul Gutierrez ${ }^{3, b}$, Veronica Petrozzi ${ }^{3, c}$, Homero Monsanto ${ }^{4, d}$, Emmanouil \\ Rampakakis $^{5, \mathrm{e}}$, Alexandra Altland ${ }^{6, f}$, Lara J. Wolfson ${ }^{6, \mathrm{~g}}$, MARVEL Peru Study Group \\ Grupo MARVEL Peru Study Group: Mao Zeta-Zeta ${ }^{7, c}$, María Eldemira Cruz-Saldarriaga ${ }^{8, h}$, \\ Raul Chuquiyauri-Haro ${ }^{9, i}$
}

\section{RESUMEN}

Objetivo: Describir las complicaciones mas frecuentes y la carga económica asociada con la varicela en el Perú. Material y métodos: Estudio multicéntrico de revisión de historias clínicas de pacientes de 1 a 14 años con diagnóstico de varicela entre 2011 y 2016. El uso de recursos de atención médica (URAM) asociados con la varicela, los costos unitarios y la pérdida de trabajo se utilizaron para estimar los costos directos e indirectos, presentados en USD (\$). Los datos de costos y URAM se combinaron con estimaciones de carga de enfermedad para calcular el costo total anual de la varicela en el Perú. Resultados: Se incluyeron un total de 179 niños con varicela (101 ambulatorios, 78 hospitalizados). Entre los pacientes ambulatorios, el 5,9\% presentó una o más complicaciones, en comparación con 96,2 \% de pacientes hospitalizados. El URAM incluyó el uso de medicamentos de venta libre $(72,3 \%$ frente a $89,7 \%$ de pacientes ambulatorios y hospitalizados, respectivamente), medicamentos con receta $(30,7 \%$ frente a $94,9 \%)$ y análisis y procedimientos $(0,0 \%$ frente a 80,8 \%). Los costos directos e indirectos por caso ambulatorio fueron $\$ 36$ y $\$ 62$ respectivamente y por caso hospitalizado fueron $\$ 548$ y $\$ 222$. El costo anual total asociado con la varicela se estimó en \$13 907 146. Conclusión: La varicela está asociada con complicaciones clínicas importantes y elevado URAM en Perú, lo que respalda la necesidad de implementación de un plan de vacunación universal.

PALABRAS CLAVE: Niño, varicela, programas de inmunización, costos y análisis de costo, costos de la atención en salud, gastos en salud. (Fuente: DeCS BIREME).

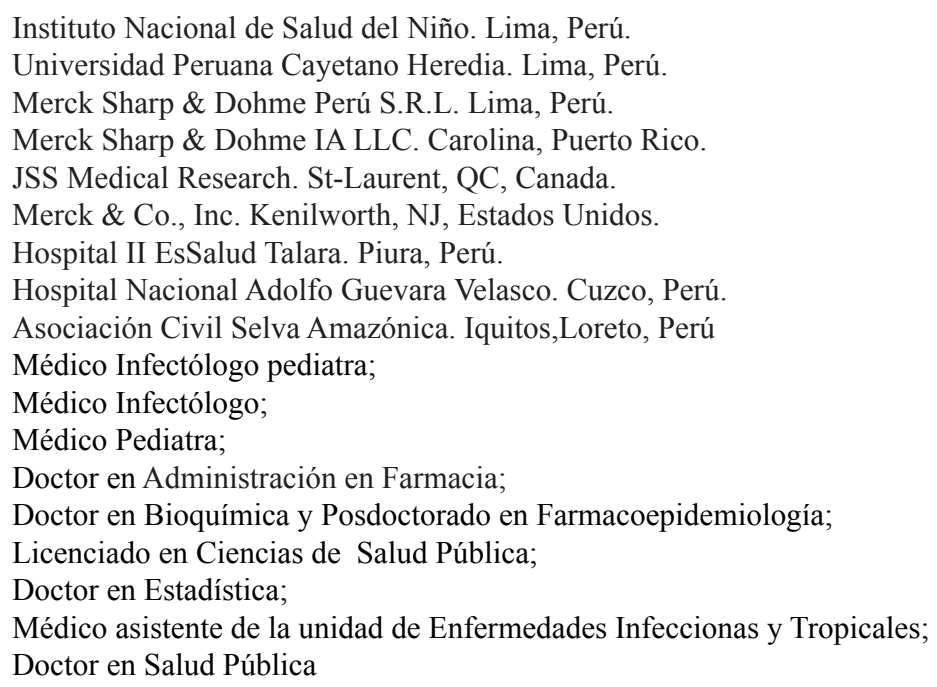




\section{SUMMARY}

Objective: The purpose of this study was to evaluate the clinical and economic burden associated with varicella in Peru. Methods: This was a multicenter, retrospective chart review study of patients aged 1-14 years with a varicella diagnosis between 2011 and 2016. Healthcare resource utilization (HCRU) associated with varicella, unit costs, and work loss were used to estimate direct and indirect costs, presented in USD (\$). The cost and HCRU data was combined with estimates of varicella disease burden to estimate the overall annual costs of management of varicella in Peru. Results: A total of 179 children with varicella (101 outpatients, 78 inpatients) were included. Among outpatients, $5.9 \%$ experienced $\geq 1$ complication, compared with $96.2 \%$ of inpatients. HCRU estimates included use of over-the-counter (OTC) medications ( $72.3 \%$ vs. $89.7 \%$ of outpatient and inpatients, respectively), prescription medications ( $30.7 \%$ vs. $94.9 \%)$, tests/procedures ( $0.0 \%$ vs. $80.8 \%)$. Among outpatients, direct and indirect costs per case were $\$ 36$ and $\$ 62$, respectively; among inpatients, respective costs were $\$ 548$ and $\$ 222$. The total annual cost associated with varicella was estimated at \$ 13907 146. Conclusion: Varicella is associated with substantial clinical complications and high HCRU in Peru, supporting the need for implementation of a routine childhood varicella vaccination plan.

KEYWORDS: Child, chickenpox, immunization programs, costs and cost analysis, health care costs. (Source: MeSH NLM).

\section{INTRODUCCIÓN}

La varicela es en general, una enfermedad autolimitada provocada por el virus de la varicelazóster (VVZ). La varicela, que típicamennte se produce durante la infancia, es muy contagiosa, se trasmite por contacto directo y por vía respiratoria (1). Una vez expuesto al VVZ, un período de incubación de 10 a 21 días precede la manifestación sintomática inicial, que puede incluir fiebre, malestar, anorexia, dolor de cabeza, y dolor abdominal (1); en 1 o 2 días, luego de los síntomas iniciales, se produce la erupción vesicular prurítica característica (1). Además, la infección por varicela puede ocasionalmente afectar gravemente los sistemas neurológico, respiratorio y dermatológico (2).

La prevención de la varicela a través de la vacunación se logra mediante la administración de vacunas contra la varicela, sea monovalente o combinada (sarampión, rubéola, parotiditis y varicela; SRPV). El uso de las vacunas contra la varicela está recomendado en niños inmunocompetentes mayores de 12 meses, y se ha demostrado, que son muy efectivas en la prevención de transmisión de la enfermedad, y tienen un alto grado de tolerabilidad (3). Sin embargo, a pesar de la disponibilidad de la vacuna, se estima que la incidencia mundial anual de la varicela es de entre 2 y 16 casos cada 1000 personas $(2,4)$, y las variaciones regionales pueden atribuirse a factores tales como edad, densidad de la población, clima y asistencia temprana a centros de cuidado a niños fuera de hogar o guarderías $(5,6)$. Con base en los datos de informes de casos regionales del Ministerio de Salud de Perú (MINSA), entre 2009 y 2014, la cantidad anual promedio de casos de varicela reportados fue 32296 (7).

El MINSA recomienda que el manejo de los síntomas de la varicela incluya descanso, ingesta adecuada de líquidos, baños diarios y corte de uñas, uso de acetaminofeno y/o antihistamínicos de primera generación, y tratamiento antivírico con aciclovir (8). La vacuna se incluyó como parte del programa de inmunización nacional en el Perú en febrero de 2018 (9).

El objetivo principal de este estudio fue describir las complicaciones mas frecuentes y la carga económica asociada con la varicela en el Perú en niños que buscaron atención médica por varicela entre 2011 y 2016.

\section{MATERIAL Y MÉTODOS}

Estudio observacional de revisión retrospectiva de historias clínicas, realizado en 9 centros de Perú (6 privados, 3 públicos). Conforme a las reglamentaciones locales, el estudio fue aprobado por el Comité de Etica en investigación del Instituto Nacional de Salud del Niño en su Sesión $\mathrm{N}^{\circ} 12$, de fecha 22 de junio de 2016. No se solicitó el consentimiento de los pacientes, ya que los datos fueron recopilados de la revisión de historias clínicas de manera anónima por los investigadores, y se identificaron solo mediante un número de paciente encriptado. Este estudio se llevó a cabo como parte de un proyecto multicéntrico de países (10-12), de conformidad con los lineamientos 
de las buenas prácticas de farmacoepidemiología (BPF).

Se seleccionaron historias de 150 pacientes como una muestra por conveniencia en los consultorios comenzando por el año más reciente y de hasta cinco años hacia atrás. La historia de cada paciente fue revisada desde la fecha de la primera visita por varicela primaria hasta la resolución de la enfermedad, o la última fecha de contacto, si la fecha de resolución no estaba disponible. Los criterios de inclusión fueron pacientes de 1 a 14 años, hospitalizados o ambulatorios con diagnóstico de varicela primaria y las fechas de diagnóstico y resolución deberían ser previas al comienzo del estudio. El grupo de pacientes ambulatorios incluía a pacientes que visitaron el consultorio del médico (médico de cabecera, médico general, pediatra o infectólogo), el consultorio del hospital o el departamento de emergencias y que no requirieron hospitalización por varicela, y los pacientes hospitalizados se definieron como aquellos que fueron inicial o eventualmente admitidos en un hospital por varicela primaria. Se intentó obtener una proporción aproximada 1:1 entre pacientes ambulatorios y hospitalizados. Los criterios de exclusión fueron pacientes con diagnóstico de herpes zoster, pacientes que hubieran recibido una vacuna contra varicela anteriormente, pacientes con diagnóstico de breakthrough varicela (varicela de brecha) o que se presentaron por un segundo diagnóstico de varicela.

Las características sociodemográficas, los antecedentes médicos, así como los parámetros de la enfermedad se extrajeron de cada historia del paciente. Las complicaciones clínicas relacionadas con la varicela que fueron evaluadas en este estudio incluyeron, infecciones de la piel y los tejidos blandos, meningitis, encefalitis, neumonía, septicemia, osteomielitis aguda, artritis séptica, cerebelitis, queratoconjuntivitis, hepatitis, nefritis, convulsiones febriles, deshidratación, dolor intenso, y trastorno de coagulación entre otras.

El uso de recursos de atención médica (URAM) para los casos de varicela y sus complicaciones incluyó: total de visitas de pacientes ambulatorios; visitas al consultorio del médico, a la sala de emergencias (SE) o al consultorio del hospital; hospitalizaciones y estadía en la unidad de cuidados intensivos (UCI); uso de medicamentos de venta libre y con receta; análisis y procedimientos realizados y consultas a profesionales de la salud no médicos. Con base en el URAM y la pérdida de ingresos de los cuidadores, los costos directos e indirectos se calcularon para los casos de varicela ambulatorios y hospitalizados, y se usaron para estimar el costo total anual debido a la varicela.

Todas las historias de los pacientes seleccionados se incluyeron en el análisis estadístico; el análisis de subgrupos se realizó para pacientes ambulatorios y hospitalizados. Se utilizó estadística descriptiva y se determinaron la media, la desviación estándar (DE), y el intervalo de confianza (IC) del 95\% de la media, para las variables continuas, y la frecuencia y el porcentaje para las variables categóricas. Se usó la transformación logarítmica para calcular con IC del 95\% cualquier medida de resultado que tuviera un bajo número de casos.

La distribución de las complicaciones de la varicela se evaluó de forma descriptiva mediante el cálculo de la frecuencia y la proporción de pacientes con al menos una complicación, así como la proporción de las complicaciones dentro de cada tipo. Otras complicaciones aparte de las enumeradas anteriormente se codificaron utilizando el Diccionario Médico para las Actividades Regulatorias (MedDRA), versión 19.0, y se informaron por clasificación de órganos y sistemas. El URAM se evaluó mediante la cantidad y la proporción de pacientes que usan cada recurso de atención médica, y la frecuencia y duración de uso, para los casos de varicela y de sus complicaciones.

El costo directo del URAM se determinó multiplicando la cantidad de recursos utilizados por paciente por el costo unitario de cada recurso, tal como se obtiene del MINSA (13), y ajustado en soles de Perú (PEN) a 2016 (14). Los costos indirectos se calcularon como la pérdida de ingresos, en días de trabajo, de los cuidadores a cargo de los niños infectados con varicela, mediante la utilización de las estadísticas de ingresos promedio nacionales (15). Se estimó la pérdida de ingresos por días de trabajo como el total de días transcurridos en el hospital o la UCI para pacientes hospitalizados durante este estudio, y 2,5 días para pacientes ambulatorios, tal como se estimó en estudios anteriores (16).

El costo de la varicela en el 2016 se calculó utilizando una estimación para América Latina donde la incidencia anual reportada en menores de 15 años fue de 42,9/1000 personas (2). Teniendo en cuenta la población pediátrica total de Perú (8 698 780) (17), se presentarían en total de 373178 casos de varicela pediátrica. Asumiendo que el $35 \%$ de estos 
buscaron atención médica (18), se calcula que 130612 casos de varicela pediátrica buscaron atención. De la cantidad anual promedio de casos de varicela reportados al sistema de vigilancia del MINSA entre 2009 y $2014(\mathrm{n}=32$ 296) (7) el 82\% de los casos se encontraban en el grupo de menores de 15 años (19) lo que representa un total de 29763 casos de varicela pediátrica (19). De estos, la cantidad total de pacientes que se hospitalizan se calculó utilizando el dato de la literatura que indica que $5,78 \%$ de los casos de varicela reportados son hospitalizados en América Latina (29 $763 \times 5,78 \%=1720$ pacientes hospitalizados). La cantidad de pacientes ambulatorios fue representada por los 128,892 casos restantes que buscaron atención (130 612 - 1,720) (2). Los costos anuales (directos + indirectos) correspondientes se calcularon en soles peruanos (PEN) al 2016 y se convirtieron a dólares estadounidenses (USD) a una tasa de cambio de 1 PEN $=0,2922$ USD (14). Se llevaron a cabo análisis estadísticos mediante el software SAS ${ }^{\circledR}$, versión 9.4 (SAS Institute Inc., Cary, NC, Estados Unidos).

\section{RESULTADOS}

Se incluyeron las historias de 179 pacientes elegibles diagnosticados con varicela primaria entre

Tabla 1. Características del paciente y la enfermedad en el momento del diagnóstico de varicela.

\begin{tabular}{|c|c|c|}
\hline & $\begin{array}{c}\text { Pacientes } \\
\text { ambulatorios } \\
(\mathbf{N}=\mathbf{1 0 1})\end{array}$ & $\begin{array}{c}\text { Pacientes } \\
\text { hospitalizados } \\
(\mathrm{N}=78)\end{array}$ \\
\hline \multicolumn{3}{|l|}{ CARACTERÍSTICAS DEL PACIENTE } \\
\hline Edad, años, media $\pm D E$ & $3,3 \pm 3,3$ & $2,4 \pm 3,4$ \\
\hline \multicolumn{3}{|l|}{ Sexo, n (\%) } \\
\hline Masculino & $58(57,4 \%)$ & $43(55,1 \%)$ \\
\hline Femenino & $43(42,6 \%)$ & $35(44,9 \%)$ \\
\hline \multicolumn{3}{|l|}{ Lugar de residencia, n (\%) } \\
\hline Urbano & $97(96,0 \%)$ & $55(70,5 \%)$ \\
\hline Rural & $4(4,0 \%)$ & $23(29,5 \%)$ \\
\hline Índice de masa corporal (IMC), $\mathrm{kg} / \mathrm{m}^{2}$, media $\pm \mathrm{DE}$ & $16,7 \pm 2,8$ & $16,8 \pm 3,8$ \\
\hline \multicolumn{3}{|l|}{ Año calendario del diagnóstico, $n(\%)$} \\
\hline 2011 & $1(1,0 \%)$ & $8(10,3 \%)$ \\
\hline 2012 & $3(3,0 \%)$ & $7(9,0 \%)$ \\
\hline 2013 & $6(5,9 \%)$ & $41(52,6 \%)$ \\
\hline 2014 & $16(15,8 \%)$ & $18(23,1 \%)$ \\
\hline 2015 & $45(44,6 \%)$ & $4(5,1 \%)$ \\
\hline 2016 & $30(29,7 \%)$ & $0(0,0 \%)$ \\
\hline Pacientes que estaban inmunocomprometidos ${ }^{\dagger}, \mathbf{n}(\%)$ & $0(0,0 \%)$ & $1(1,3 \%)$ \\
\hline \multicolumn{3}{|l|}{ CARACTERÍSTICAS DE LA ENFERMEDAD } \\
\hline \multicolumn{3}{|c|}{ Número máximo de lesiones cutáneas durante la erupción, n (\%) } \\
\hline$<50$ & $56(55,4 \%)$ & $1(1,3 \%)$ \\
\hline $50-249$ & $35(34,7 \%)$ & $7(9,0 \%)$ \\
\hline $250-500$ & $9(8,9 \%)$ & $50(64,1 \%)$ \\
\hline$>500$ & $1(1,0 \%)$ & $20(25,6 \%)$ \\
\hline
\end{tabular}




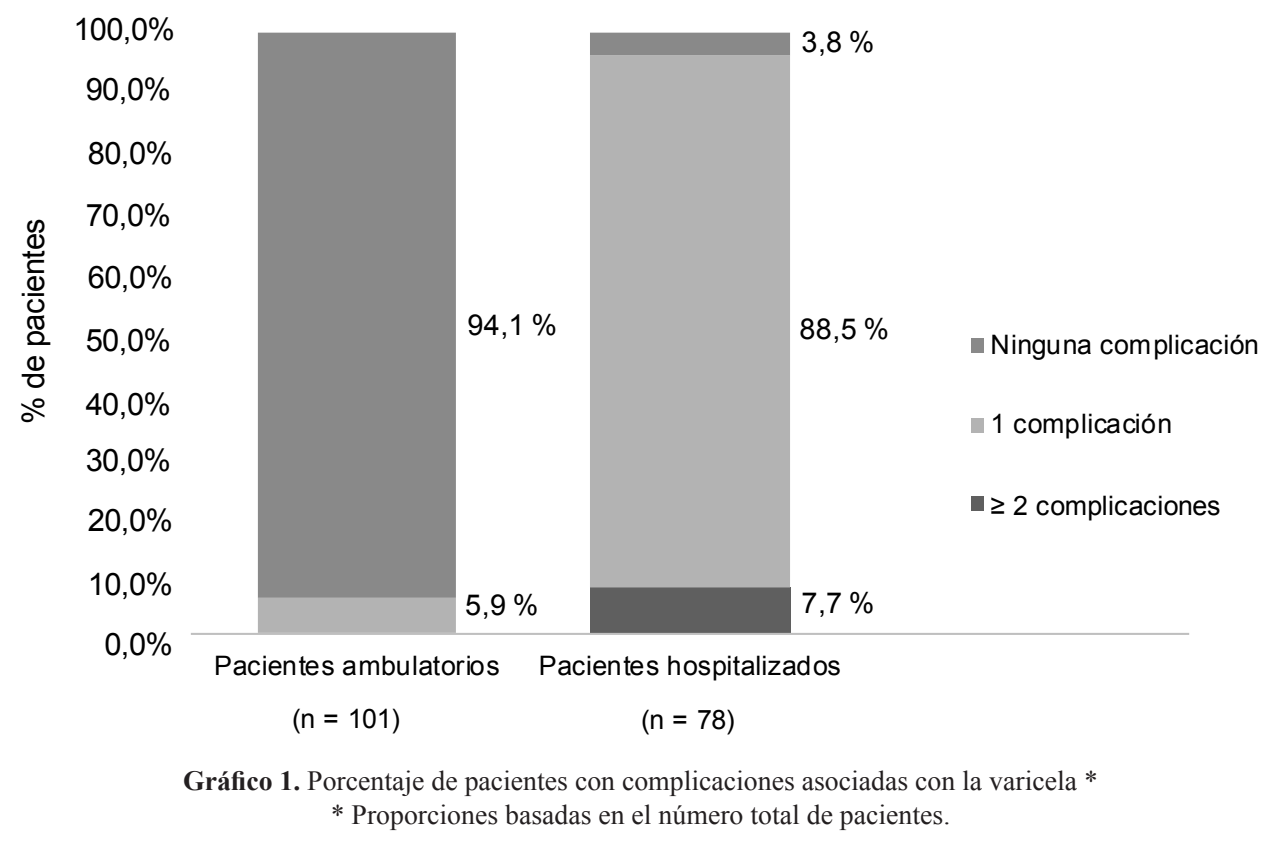

Tabla 2. Uso de recursos de atención médica asociados con la varicela.

\begin{tabular}{|c|c|c|c|c|}
\hline \multirow[t]{2}{*}{ Tipo de URAM } & \multicolumn{2}{|c|}{$\begin{array}{l}\text { Pacientes ambulatorios } \\
\qquad(\mathrm{N}=101)\end{array}$} & \multicolumn{2}{|c|}{$\begin{array}{c}\text { Pacientes hospitalizados } \\
\qquad(\mathrm{N}=78)\end{array}$} \\
\hline & $\%$ & $\begin{array}{c}\text { Media } \\
(\text { IC del } 95 \%)^{\dagger} \\
\end{array}$ & $\%$ & $\begin{array}{c}\text { Media } \\
(\text { IC del } 95 \%)^{\dagger} \\
\end{array}$ \\
\hline Visitas al consultorio médico & $84,2 \%$ & $1,1(0,9 ; 1,3)$ & $14,1 \%$ & $1,0(0,6 ; 1,8)$ \\
\hline Visitas a la SE & $13,9 \%$ & $1,0(0,6 ; 1,7)$ & $96,2 \%$ & $1,0(0,8 ; 1,3)$ \\
\hline Visitas a consultas ambulatorias de hospitales & $24,8 \%$ & $1,1(0,8,1,6)$ & $3,8 \%$ & $1,0(0,3 ; 3,1)$ \\
\hline Visitas ambulatorias totales & $100 \%$ & $1,3(1,1 ; 1,6)$ & $100 \%$ & $1,1(0,9 ; 1,4)$ \\
\hline Hospitalización ${ }^{\S}$ & N/A & $\mathrm{N} / \mathrm{A}$ & $100 \%$ & $6,8(5,8 ; 8,0)$ \\
\hline Estancia en la UCI & $\mathrm{N} / \mathrm{A}$ & N/A & $1,3 \%$ & $7,0(\mathrm{~N} / \mathrm{C})$ \\
\hline Medicamentos con receta & $30,7 \%$ & $1,2(0,8 ; 1,6)$ & $94,9 \%$ & $2,3(1,9 ; 2,6)$ \\
\hline Medicamentos de venta libre & $72,3 \%$ & $1,6(1,3 ; 1,9)$ & $89,7 \%$ & $1,9(1,6 ; 2,2)$ \\
\hline Análisis y procedimientos & $0,0 \%$ & N/A & $80,8 \%$ & $2.9(2,5 ; 3,4)$ \\
\hline Consultas a otros profesionales de la salud & $0,0 \%$ & $\mathrm{~N} / \mathrm{A}$ & $39,7 \%$ & $1,5(1,1 ; 1,9)$ \\
\hline \multicolumn{5}{|c|}{ 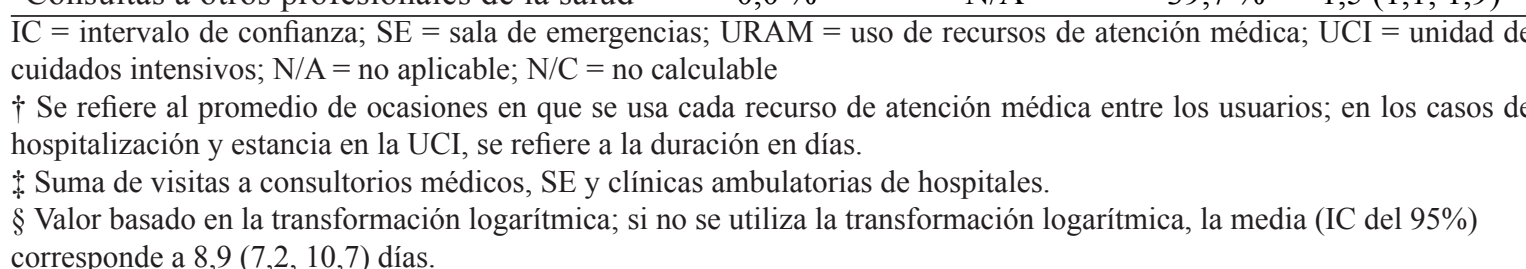 } \\
\hline
\end{tabular}

2011 y 2016; las características iniciales se resumen en la tabla 1.

Las complicaciones de la varicela fueron más frecuentes en los pacientes hospitalizados. E1 88,5\% de los pacientes hospitalizados $(n=69 / 78)$ presentó una complicación de varicela, y el 7,7\% $(n=6 / 78)$ presentó $\geq 2$; en comparación de los pacientes ambulatorios $5,9 \%(n=6 / 101)$ informaron solo una complicación de la varicela (gráfico 1). Todas las complicaciones de la varicela en pacientes ambulatorios $(n=6 / 6)$ se identificaron como infección de piel y tejido blando. De las complicaciones informadas en pacientes hospitalizados, la mayoría fueron infección de la piel 
Tabla 3. Costos unitarios - (2016 USD)† para recursos de atención médica.

\begin{tabular}{|c|c|}
\hline & Costo promedio * \\
\hline \multicolumn{2}{|l|}{ Recurso de atención médica } \\
\hline Visitas al consultorio médico & USD $\quad 5,9$ \\
\hline Visitas a la SE & USD 21,8 \\
\hline Visitas clínicas ambulatorias de hospitales & USD $\quad 5,9$ \\
\hline Día de hospitalización & USD $\quad 49,5$ \\
\hline Día de estancia en la UCI & USD 276,9 \\
\hline \multicolumn{2}{|c|}{$\begin{array}{l}\text { PEN = Sol peruano; } \mathrm{SE}=\text { sala de emergencias; UCI = unidad de cuidados intensivos; USI } \\
=\text { dólares estadounidenses. } \\
\text { *Fuente de costos obtenidos del MINSA de Perú (13) y convertidos a PEN de } 2016 \text { (14). }\end{array}$} \\
\hline
\end{tabular}

Tabla 4. Costo (USD) por caso pediátrico de varicela

\begin{tabular}{|c|c|c|c|c|}
\hline & \multicolumn{2}{|c|}{ Pacientes ambulatorios $(\mathrm{N}=101)$} & \multicolumn{2}{|c|}{$\begin{array}{c}\text { Pacientes hospitalizados } \\
(\mathbf{N}=78)\end{array}$} \\
\hline & $\begin{array}{l}\text { Costo } \\
\text { promedio } \\
\text { (US\$) }\end{array}$ & IC del $95 \%$ & $\begin{array}{l}\text { Costo } \\
\text { promedio }^{\dagger} \\
\text { (US\$) }\end{array}$ & IC del $95 \%$ \\
\hline \multicolumn{5}{|l|}{ COSTOS DIRECTOS } \\
\hline Visitas al consultorio médico & 5,5 & $(50 ; 6,1)$ & 0,8 & $(0,4 ; 1,3)$ \\
\hline Visitas a la SE & 3 & $(1,5 ; 4,5)$ & 21 & $(20,0 ; 21,9)$ \\
\hline Visitas clínicas ambulatorias de hospitales & 1,6 & $(1,0 ; 2,2)$ & 0,2 & $(0,0 ; 0,5)$ \\
\hline Hospitalización & $\mathrm{N} / \mathrm{A}$ & N/A & 442,4 & $(356,2 ; 528,5)$ \\
\hline Estancia en la UCI & $\mathrm{N} / \mathrm{A}$ & $\mathrm{N} / \mathrm{A}$ & 24,8 & $(0,0 ; 74,3)$ \\
\hline Medicamentos con receta & 25,3 & $(2,1 ; 48,6)$ & 40,4 & $(27,7 ; 53,0)$ \\
\hline Medicamentos de venta libre & 0 & $(1,5 ; 5,7)$ & 0,1 & $(0,0 ; 0,1)$ \\
\hline Análisis y procedimientos & 0 & $\mathrm{~N} / \mathrm{C}$ & 14,1 & $(11,0 ; 17,2)$ \\
\hline Consultas a otros profesionales de la salud & 0 & $\mathrm{~N} / \mathrm{C}$ & 4 & $(2,9 ; 5,2)$ \\
\hline Costos directos totales & 35,5 & $(11,9 ; 59,1)$ & $\mathbf{5 4 7 , 8}$ & $(438,3 ; 657,3)$ \\
\hline \multicolumn{5}{|l|}{ COSTOS INDIRECTOS } \\
\hline Trabajo que pierden los cuidadores & 62,1 & $\mathrm{~N} / \mathrm{C}$ & 222,1 & $(178,8 ; 265,3)$ \\
\hline TOTAL & 97,6 & $(74,1 ; 121,2)$ & 769,9 & $(621,0 ; 918,8)$ \\
\hline
\end{tabular}

y del tejido blando $(75,3 \%)$, seguido de neumonía $(6,2 \%)$, complicaciones neurológicas $(6,2 \%$ : incluye encefalitis $3,7 \%$ y cerebelitis $2,5 \%$ ). El restante de las complicaciones $(12,3 \%)$ fueron artritis séptica $(2,5 \%)$, trastornos musculo esqueléticos y del tejido conectivo $(2,5 \%)$, sepsis $(1,2 \%)$, trastorno de coagulación $(1,2 \%)$, deshidratación $(1,2 \%)$, trastornos renales y urinarios $(1,2 \%)$, y trastornos de la piel y del tejido subcutáneo $(1,2 \%)$ y otros $(1,2 \%)$.
En la tabla 2 se resume el URAM asociado con la varicela en los pacientes ambulatorios y los pacientes hospitalizados. De la cantidad total de medicamentos de venta libre informada, los más frecuentes informados fueron acetaminofeno/ ibuprofeno (36\%) y antihistamínicos orales (36\%). Entre los medicamentos con receta, los antibióticos representaron $81 \%$ del total de recetas, predominando oxacilina $(21 \%)$, cefalexina $(15 \%)$ y clindamicina 
Tabla 5. Costos anuales estimados (2016) (USD) para niños con varicela en Perú

\begin{tabular}{lcc}
\hline & $\begin{array}{c}\text { Costo anual } \\
\text { (USD) }\end{array}$ & $\begin{array}{c}\text { (\%) de los costos } \\
\text { directos totales }\end{array}$ \\
\hline COSTOS DIRECTOS & & \\
Visitas al consultorio médico & 710882,0 & 12,9 \\
Visitas a la SE & 425893,7 & 7,7 \\
Visitas clínicas ambulatorias de hospitales & 204892,0 & 3,8 \\
Hospitalización & 760859,2 & 13,8 \\
Estancia en la UCI & 42740,3 & 0,8 \\
Medicamentos con receta & 3336561,6 & 60,5 \\
Medicamentos de venta libre & 2007,6 & 0,0 \\
Análisis y procedimientos & 24274,4 & 0,4 \\
Consultas a otros profesionales de la salud & 6910,6 & 0,1 \\
Costos directos totales & 5517125,8 & 100,0 \\
COSTOS INDIRECTOS & & \\
Trabajo que pierden los cuidadores & 8390020,4 & N/A \\
TOTAL & 13907146,2 & N/A \\
\hline SE
\end{tabular}

$\mathrm{SE}=$ sala de emergencias; $\mathrm{UCI}=$ unidad de cuidados intensivos; N/A = no aplicable; $\mathrm{USD}=$ dólares estadounidenses

${ }^{\dagger}$ Las estimaciones de costos por caso de varicela aplicadas derivan de la Tabla 4.

(15\%). El consumo de antibióticos en pacientes hospitalizados fue de 14,8 $\pm 11,2$ días, y en pacientes ambulatorios fue de $6,5 \pm 2,6$ días.

La tabla 3 muestra el costo unitario de los recursos de atención médica clave utilizados en el cálculo de costos directos por caso de varicela mostrados en la tabla 4, para pacientes ambulatorios y pacientes hospitalizados por tipo de recurso utilizado en el cual se muestra que el costo total (directo + indirecto) promedio (DE) estimado por caso de varicela fue de 97,6 USD $(74,1 ; 121,2)$ y 769,9 USD $(621,0$; 918,8 ), para pacientes ambulatorios y hospitalizados, respectivamente.

El costo anual estimado asociado con la infección por varicela pediátrica en 2016 para Perú se presenta en la tabla 5. De un total de 130612 casos de varicela estimados que buscaron atención médica (1720 pacientes hospitalizados y 128892 pacientes ambulatorios), se estimó que los costos directos e indirectos totales fueron de 5517 125,8 USD y 8390 020,4 USD, respectivamente, para un total de 13907 146,2 USD.

\section{DISCUSIÓN}

Los resultados de este estudio retrospectivo demuestran que la infección por varicela pediátrica en Perú se asocia con una importante carga clínica y de atención médica en pacientes ambulatorios y hospitalizados. Además de los costos directos sanitarios, desde la perspectiva social, los costos indirectos imparten una carga financiera considerable en los costos totales ocasionados por la enfermedad. Alrededor del $60 \%$ de los costos totales asociados a la varicela fueron atribuidos a pérdidas de días de trabajo de los cuidadores.

La presencia de lesiones de varicela puede facilitar el ingreso de otros patógenos bacterianos, así la población pediátrica representa un grupo de riesgo para el desarrollo de complicaciones de la piel. Estas complicaciones incluyen impétigo, celulitis, absceso o fascitis, todas las cuales requieren de hospitalización y tratamiento antimicrobiano (20). Un hallazgo importante del presente estudio está relacionado con la carga de uso de antibióticos, que se encontró que constituye más del $80 \%$ de todos los medicamentos con receta administrados. La resistencia antimicrobiana, que está asociada con complicaciones más graves, estadías mas prolongadas en el hospital y tasas de mortalidad más altas; es exacerbada por la prescripción de antibióticos, un fenómeno observado en atención primaria y entornos hospitalarios (21).

Las tasas de complicaciones descritas en este estudio son similares a las obtenidas en un estudio 
que evalúa la carga de la enfermedad y el costo de la varicela en pacientes pediátricos hospitalizados en el Instituto Nacional de Salud del Niño (INSN) en 2013 (22). El estudio encontró que el $72,4 \%$ de los pacientes presentaron infecciones de la piel y del tejido blando, seguido por complicaciones del sistema nervioso central $(13,4 \%$ que incluye cerebelitis, encefalitis, meningitis y "otras") e infecciones respiratorias (11,1\%, incluidas neumonías y "otras"). Otro estudio peruano que evaluó la varicela pediátrica en el INSN entre 2001 y 2011 informó una tasa levemente más baja de infección de piel $(42,6 \%)$, mientras que la neumonía y las complicaciones neurológicas fueron informadas por el $12,3 \%$ y $5,7 \%$ de los pacientes, respectivamente (23).

La proporción más alta de complicaciones observada de pacientes hospitalizados (96,2\%) frente a pacientes ambulatorios (5,9\%) también es consistente con los datos anteriormente publicados. Estudios realizados en Italia y Alemania informaron tasas de complicaciones en pacientes ambulatorios del $3,5 \%$ y $5,9 \%(24,25)$, mientras que en un estudio en el Perú, mostró que el $68,5 \%$ de los pacientes hospitalizados informaron complicaciones (23). Mientras que la tasa informada de complicaciones de pacientes hospitalizados en este estudio es más alta, no se puede determinar si esto se debe a la incidencia de una enfermedad más grave captada en el estudio actual, o es producto de diferencias metodológicas en la recolección y el informe de complicaciones.

Las estimaciones de costos por paciente hospitalizado pediátrico para varicela se han determinado anteriormente en un estudio publicado, donde el costo directo total estimado por paciente hospitalizado tratado en el INSN fue de 2481,02 PEN (725 USD por paciente) (22), en comparación con los aproximadamente 550 USD estimados por paciente hospitalizado en el estudio actual. Además, esta es la primera publicación de los costos anuales totales por varicela en Perú, y es comparable con las estimaciones proporcionadas en análisis de costo-efectividad anteriormente publicadas en Latinoamérica. Se estimó que el costo total anual de la varicela en Brasil fue de R\$ 27378957 para la sociedad en el 2004, y 17588725 USD en $2016(26,27)$, lo que refleja 879095 visitas de pacientes ambulatorios y 4507 hospitalizaciones. El costo directo a 30 años de la varicela en Colombia fue estimado en 89 millones de USD en 2008 ( 3 millones de USD anualmente), lo que refleja 490138 consultas y 945 hospitalizaciones (18). Estos resultados pueden compararse con el estudio actual: mediante el URAM promedio calculado por paciente (tabla 2), extrapolado a 128892 pacientes ambulatorios y 1720 pacientes hospitalizados, con un costo directo total anual de aproximadamente 5,5 millones de USD (tabla 5). Dadas las diferencias entre los países en el comportamiento de búsqueda de atención médica, la precisión de los datos de vigilancia de la varicela, las políticas gubernamentales y los algoritmos de tratamiento, se debe tener precaución a la hora de obtener paralelos entre los estudios en diferentes países.

En las últimas décadas, Perú ha observado un aumento en la urbanización y densidad de la población (28). También se han observado tasas en aumento en asistencia preescolar y secundaria: en 2015, se informó que más del 99\% de los niños había ingresado en programas educativos 1 año antes del inicio de la escuela primaria, en comparación con un 75\% en 1999 (29). Las tasas de asistencia en educación secundaria también aumentaron del $62 \%$ en 1999 a casi el $78 \%$ en 2015 (29). Dado que se han identificado la densidad de la población y la asistencia temprana fuera del hogar en niños pequeños como factores que afectan la trasmisión de la varicela $(5,6)$, este cambio observado en las características sociodemográficas de Perú puede fomentar ambientes en los que la circulación y trasmisión de una enfermedad infecciosa, como varicela, es más intensa.

En febrero de 2018, Perú se unió a otros países latinoamericanos en incluir la vacuna contra la varicela en su programa de inmunización nacional $(9,30)$. En Uruguay, un estudio hecho después de 6 años de la introducción de la vacuna contra varicela en su calendario nacional a los 12 meses de edad con altas tasas sostenidas de cobertura vacunal, demostró reducción de $81 \%$ y $87 \%$ de las hospitalizaciones y de las consultas de pacientes ambulatorios relacionadas a varicela, respectivamente (31). De forma similar, Costa Rica mostró una reducción del 73,8\% de casos reportados de varicela y una reducción del $85,9 \%$ de hospitalizaciones en la población general después de 8 años de la introducción de un programa universal de vacuna de varicela con una dosis en niños de 15 meses de edad (32).

Las limitaciones de este estudio incluyen la revisión retrospectiva de historias clínicas, ya que solo puede haberse captado una sección transversal de la atención de los pacientes, lo que podría haber derivado en una subestimación del URAM asociado. Asimismo, el posible sesgo en la selección de casos que buscan 
atención médica puede haber exagerado la estimación de la carga asociada con cada caso de varicela. Sin embargo, hemos intentado representar esto en la extrapolación del costo anual nacional. Además, debido a la falta de datos locales de Perú, algunos supuestos en el modelo de estimación de costos se basaron en datos específicos de América Latina cuando no estaban disponibles los datos locales para Perú. Otra limitación es que no se realizó una evaluación de los gastos que el paciente desembolsa de su bolsillo, y se realizó solo una evaluación limitada del costo indirecto debido a pérdida de trabajo. La incidencia anual estimada de la varicela y los costos asociados se basaron en varios supuestos, y la cantidad de casos informados se basó en el sistema de reporte de casos regional del MINSA, así como la cantidad de contactos de atención médica debido a una infección por varicela, que es probable que sea subestimado. Tampoco se captó todo el impacto social, ya que las estimaciones de costos no consideraron el manejo de brotes de varicela en entornos hospitalarios. Finalmente, el tamaño relativamente pequeño de la muestra del estudio y el pequeño número de centros participantes pueden reducir la validez externa de los hallazgos. Entre estos hallazgos, se encontró que la población pediátrica con varicela fue predominantemente de sexo masculino, diferente de lo encontrado en la literatura que muestra incidencia similar entre los generos.

Este estudio mostró que la carga económica de enfermedad asociada con la varicela en Perú, en niños que buscaron atención médica por varicela entre 2011 y 2016, estuvo asociada con altas tasas de complicaciones de pacientes hospitalizados, carga económica en los cuidadores y elevado URAM, más específicamente con respecto al uso de antibióticos. Con base en los datos generados sobre las estimaciones locales del impacto de la varicela en los pacientes, sus cuidadores y el sistema sanitario, los resultados presentados aquí respaldan la necesidad de vacunación contra la varicela infantil de rutina para reducir la carga asociada a esta enfermedad en Perú.

\section{Declaración de financiamiento y de conflictos de intereses:}

RG, VP, HM, AA y LJW son empleados de Merck Sharp \& Dohme Corp., una subsidiaria de Merck \& Co., Inc., Kenilworth, NJ, EE. UU., que podrían poseer acciones y/o mantener opciones sobre acciones en la compañía. MC ha recibido apoyo de investigación de Sanofi Pasteur, Merck \& Co., Inc., Kenilworth, NJ, EE. UU. y Pfizer, y brindó servicios como asesor contratado a Merck \& Co., Inc., Kenilworth, NJ, EE. UU. ER es un empleado de JSS Medical Research, que fue contratada por Merck \& Co., Inc., Kenilworth, NJ, EE. UU., para llevar a cabo el estudio y proporcionar apoyo para la redacción de este escrito. MECS se ha desempeñado como conferencista pago para Merck \& Co., Inc., Kenilworth, NJ, EE. UU. MZZ y RCH no han informado ningún conflicto de interés. Este estudio fue financiado por Merck \& Co., Inc., Kenilworth, NJ, EE. UU.

\section{Contribución de autoría:}

HM, ER, MEC y LJW conceptualizaron el diseño del estudio. MZZ, MECS, RCH y MC adquirieron los datos. VP, RG, AA, MEC y AJW asistieron en el análisis y la interpretación de los datos; ER y LW diseñaron los análisis estadísticos y ER los llevó a cabo. ER, AA y LJW redactaron el escrito. Todos los autores revisaron las versiones finales preliminares del escrito, e hicieron sus aportes en relación con la estructura y el contenido. La versión final del escrito presentada para su publicación fue revisada y aceptada por todos los autores.

\section{Correspondencia}

\section{Veronica Petrozzi}

Merck Sharp \& Dohme Perú SRL

Av. Circunvalación del Club Golf los Incas 134, Torre 2, Oficina 1901

Surco, Lima, Perú.

Correo electrónico: veronica.petrozzi@merck.com

\section{REFERENCIAS BIBLIOGRÁFICAS}

1. WHO. Varicella and herpes zoster vaccines: WHO position paper, June 2014. Relev Epidemiol Hebd. 2014; 89(25):265-87.

2. Bardach A, Cafferata ML, Klein K, Cormick G, Gibbons L, Ruvinsky S. Incidence and use of resources for chickenpox and herpes zoster in Latin America and the Caribbean--a systematic review and meta-analysis. Pediatr Infect Dis J. 2012; 31(12):1263-8.

3. Wutzler P, Bonanni P, Burgess M, Gershon A, Safadi MA, Casabona G. Varicella vaccination - the global experience. Expert Rev Vaccines. 2017; 16(8):83343.

4. Heininger U, Seward JF. Varicella. Lancet. 2006; 368(9544):1365-76.

5. Lolekha S, Tanthiphabha W, Sornchai P, et al. Effect of climatic factors and population density on varicella zoster virus epidemiology within a tropical country. 
Am J Trop Med Hyg. 2001; 64(3-4):131-6.

6. Yawn BP, Yawn RA, Lydick E. Community impact of childhood varicella infections. J Pediatr. 1997; 130(5):759-65.

7. Ministerio de Salud de Perú. Casos de Varicela por Meses del año 2009 al 2016* en 06 DIRESAs del Perú. Lima: Centro Nacional de Epidemiologia, Prevencion y Control de Enfermedades; 2016. (Fecha de acceso 16 de julio del 2018). Disponible en: http://www.dge. gob.pe/portal/docs/vigilancia/sala/2016/SE42/ varicela.pdf

8. Ministerio de Salud. Guía de Práctica Clínica de Varicela en Niños. Lima: Instituto Nacional de Salud del Niño;2018. (Fecha de acceso 16 de julio del 2018). Disponible en: http://bibliotecavirtual.insnsb. gob.pe/guia-de-practica-clinica-de-varicela-en-ninos/

9. Ministerio de Salud . Norma Técnica de Salud que establece el Esquema Nacional de Vacunación: Lima: Ministerio de Salud; 2018. (Fecha de acceso el 16 de julio del 2018). Disponible en: http://www.MINSA. gob.pe/index.asp?op=10

10. Giglio N, Monsanto H, Rampakakis E, Yang HK, Kuter BJ, Wolfson LJ. Economic burden of varicella in children 1-12 years of age in Argentina, 20092014. J Med Econ. 2018; 21(4):416-24.

11. Wysocki J, Malecka I, Stryczynska-Kazubska J, Rampakakis E, Kuter B, Wolfson LJ. Varicella in Poland: economic burden in children 1-12 years of age in Poland, 2010-2015. BMC Public Health. 2018; 18(1):410.

12. Meszner Z, Molnar Z, Rampakakis E, Yang HK, Kuter BJ, Wolfson LJ. Economic burden of varicella in children 1-12 Years of age in Hungary, 2011-2015. BMC Infect Dis. 2017; 17(1):495.

13. Ministerio de Salud. Costeo y actualizacion del maestro de procedimientos hospitalarios del SIS 2013. Lima: Programa de Apoyo a la Reforma del Sector Salud; 2018. (Fecha de acceso 16 de julio del 2018). Disponible en: http://www.parsalud. gob.pe/asistencia_tecnica/aseguramiento/3Costeo_ SIS/CosteoProcedimientosHospitalerios_SIS.pdf

14. Instituto Nacional de Estadistica e Informatica. Índice de precios al consumidor por departamento 2017. Lima: Instituto Nacional de Estadistica e Informatica; 2018. (Fecha de acceso 16 de julio del 2018). Disponible en: http://iinei.inei.gob.pe/indices/.

15. Organization for economic co-operation and development. OECD.stat. 2015. Paris: Organization for economic co-operation and development; 2018. (Fecha de acceso 16 de julio del 2018). Disponible en: https://stats.oecd.org/

16. Ginsberg GM, Somekh E. Cost containment analysis of childhood vaccination against varicella in Israel. J Infect. 2004; 48(2):119-33.

17. World Health Organization. Vaccine-preventable diseases: monitoring system. 2017 global summary.
Geneva: World Health Organization; 2017. (Fecha de acceso 16 de julio del 2018). Disponible en: http:// apps.who.int/immunization_monitoring/ globalsummary/countries?countrycriteria $\% 5 \mathrm{Bcountr}$ $\mathrm{y} \% 5 \mathrm{D} \% 5 \mathrm{~B} \% 5 \mathrm{D}=\mathrm{PER} \&$ commit $=\mathrm{OK}$

18. Paternina-Caicedo A, De la Hoz-Restrepo F, GamboaGaray O, Castaneda-Orjuela C, Velandia-Gonzalez M, Alvis-Guzman N. How cost effective is universal varicella vaccination in developing countries? A case-study from Colombia. Vaccine. 2013; 31(2):4029.

19. Centro Nacional de Epidemiologia, Prevención y Control de Enfermedades, Ministerio de Salud. Sala de Situación Salud Perú: Reportes 2009-2016. Lima: Ministerio de Salud; 2017.

20. Bozzola E, Bozzola M, Krzysztofiak A, Tozzi AE, El Hachem M, Villani A. Varicella skin complications in childhood: A case series and a systematic review of the literature. Int J Mol Sci. 2016; 17(5):0-0.

21. Llor C, Bjerrum L. Antimicrobial resistance: risk associated with antibiotic overuse and initiatives to reduce the problem. Ther Adv drug Saf. 2014; 5(6):229-41.

22. Castillo ME, Del-Aguila O. Direct costs and clinicalepidemiological characteristics of hospitalized patients with chickenpox in 2013 at Instituto Nacional de Salud del Niño. Rev Peru Pediatr. 2016; 68(2):0-0.

23. Miranda-Choque E, Candela-Herrera J, Diaz-Pera J, Farfan-Ramos S, Munoz-Junes EM, EscalanteSantivanez IR. Complicated chickenpox in a national pediatric Peruvian hospital, 2001-2011. Rev Peru Med Exp Salud Publica. 2013; 30(1):45-8.

24. Fornaro P, Gandini F, Marin M, et al. Epidemiology and cost analysis of varicella in Italy: results of a sentinel study in the pediatric practice. Italian Sentinel Group on Pediatric Infectious Diseases. Pediatr Infect Dis J. 1999; 18(5):414-9.

25. Wagenpfeil S, Neiss A, Banz K, Wutzler P. Empirical data on the varicella situation in Germany for vaccination decisions. Clin Microbiol Infect. 2004; 10(5):425-30.

26. Valentim J, Sartori AMC, de Soarez PC, Amaku M, Azevedo RS, Novaes HMD. Cost-effectiveness analysis of universal childhood vaccination against varicella in Brazil. Vaccine. 2008; 26(49):6281-91.

27. StatBureau. Brazilian Inflation Calculators. Washington: StatBureau; 2004. (Fecha de acceso 16 de julio del 2018). Disponible en: https:// www.statbureau.org/en/brazil/inflationcalculators?dateBack $=2004-1-1 \&$ date To $=2016-1$ 1\&amount $=27378957.00$

28. Centro Nacional de Epidemiologia, Prevención y Control de Enfermedades. Sala situacional para el Análisis de Situación de Salud - SE 41-2018. Lima: Centro Nacional de Epidemiologia, Prevención y Control de Enfermedades; 2018. (Fecha de acceso 16 
de julio del 2018). Disponible en: http://www.dge. gob.pe/portal/index.php?option=com_content\&view $=$ article\&id $=14 \&$ Itemid $=154$

29. UNESCO; Institute for Statistics. Education. Participation; Enrolment by level of education, Peru. Washington: UNESCO; 2018. (Fecha de acceso 16 de julio del 2018). Disponible en: http://data.uis. unesco.org/\#

30. Parellada CI, Perez ME, Massoc MA, et al. A perspective on the introduction of public varicella vaccination in Latin America and Caribbean. Cancun,
Mexico: SLIPE Congress; 2017 Nov 9-11.

31. Quian J, Ruttimann R, Romero C, et al. Impact of universal varicella vaccination on 1-year-olds in Uruguay: 1997-2005. Arch Dis Child. 2008; 93(10):845-50.

32. Avila-Aguero ML, Ulloa-Gutierrez R, CamachoBadilla K, Soriano-Fallas A, Arroba-Tijerino R, Morice-Trejos A. Varicella prevention in Costa Rica: impact of a one-dose schedule universal vaccination. Expert Rev Vaccines. 2017; 16(3):229-34.

Recibido: $20 / 11 / 2018$

Aceptado: 28/03/2019 